\title{
Cattle tick vaccine researchers join forces in CATVAC
}

\author{
Theo Schetters ${ }^{1,2^{*}}$, Richard Bishop ${ }^{3}$, Michael Crampton ${ }^{4}$, Petr Kopáček ${ }^{5}$, Alicja Lew-Tabor ${ }^{6,7}$, Christine Maritz-Olivier ${ }^{8}$, \\ Robert Miller ${ }^{9}$, Juan Mosqueda ${ }^{10}$, Joaquín Patarroyo ${ }^{11}$, Manuel Rodriguez-Valle ${ }^{6}$, Glen A. Scoles ${ }^{12}$ \\ and José de la Fuente $e^{13,14}$
}

\begin{abstract}
A meeting sponsored by the Bill \& Melinda Gates Foundation was held at the Avanti Hotel, Mohammedia, Morocco, July 14-15, 2015. The meeting resulted in the formation of the Cattle Tick Vaccine Consortium (CATVAC).
\end{abstract}

Keywords: CATVAC, Vaccine, Cattle, Tick, Rhipicephalus microplus

\section{Control of cattle tick infestations: an urgent need in Africa}

According to results from a continent-wide survey in Africa conducted in 2014-2015, ticks and tick-transmitted diseases, gastrointestinal helminth infections, and Peste des Petits Ruminants (PPR) are the most important diseases that affect the livelihood and development of communities in rural Africa that depend on livestock [1]. Extensive use of acaricides to control tick infestation has led to the selection for strains that are resistant against a number of these drugs [2]. This is particularly evident for the cattle tick Rhipicephalus microplus due to the fact that the different tick stages develop on a single host. Recently, it became apparent that this tick species is now spreading over even larger areas of the African continent, thus posing an immediate and further threat to livestock. Additional control methods are urgently required to limit tick populations [3].

Ever since it was shown that blood-feeding ectoparasites were affected when feeding on hosts that had been vaccinated with crude extracts of these arthropods, researchers aimed at developing effective vaccines $[4,5]$. These efforts have met with some success in cattle tick vaccine research, but none of the vaccines appeared effective enough to sufficiently control tick infestation or transmission of the pathogens carried by them [5].

\footnotetext{
* Correspondence: th.schetters@protactivity.com

'ProtActivity R\&D, Cuijk, The Netherlands

${ }^{2}$ ClinVet International, Bloemfontein, South-Africa

Full list of author information is available at the end of the article
}

Until now, there have been few attempts to study the effects of combinations of the partially protective tick antigens, which could potentially enhance vaccine efficacy against tick infestations and pathogen infection and transmission. In order to establish a closer collaboration between research groups that work on tick vaccines, a two-day meeting sponsored by the Bill \& Melinda Gates Foundation was held on July 14-15 ${ }^{\text {th }}, 2015$ at the Avanti Hotel in Mohammedia, Morocco. Key players in tick vaccine research from the different continents were invited to give an update on their research and to participate in the discussions. The purpose of the meeting was to exchange scientific knowledge, update the tick vaccine research, and select possible combinations of tick antigens that hold promise as an effective tick vaccine against cattle ticks, R. microplus. At the meeting it was also decided to establish a formal collaboration among the groups to facilitate exchange of antigens and evaluation of candidate vaccine formulations in standardized experimental models. This collaboration resulted in the formation of the Cattle Tick Vaccine Consortium (CATVAC; Fig. 1).

\section{Cattle tick vaccination studies Bm86/Bm95}

The only recombinant tick vaccines on the market contain $100 \mu \mathrm{g}$ of the immunoprotective midgut antigen Bm86 of R. microplus produced in Pichia pastoris and are formulated in water-in-oil adjuvants. On average, the level of protection obtained as judged by reduction of the number of engorged females after tick infestation is approximately $50 \%$, but this percentage depends on the tick species and 


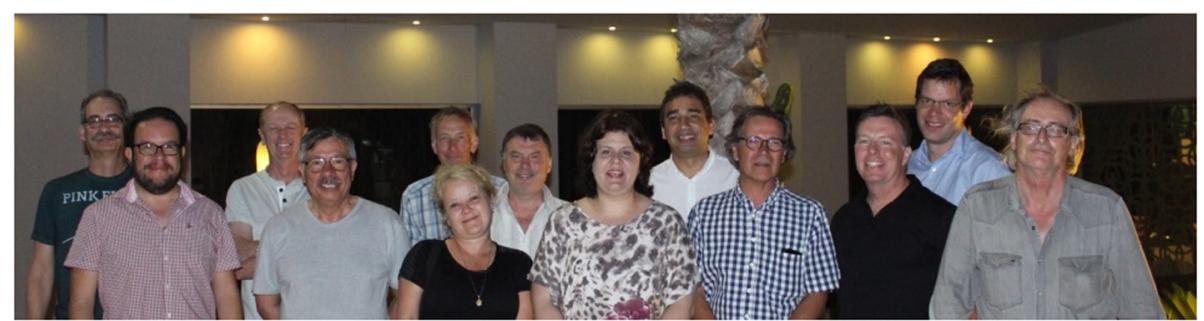

Fig. 1 The participants of the official launch meeting of CATVAC. G.A. Scoles not on the picture. This meeting was held in Mohammedia, Morocco, July 14-15, 2015

strain involved [6]. Moreover, post-engorgement female mortality and effects on female fertility may reduce the number of infective larvae by up to a maximum of $90 \%$, depending on tick strains [6]. Additionally, Peter Willadsen indicated that Bm86 produced in Escherichia coli and baculovirus has also been successfully used as vaccine antigens [7].

\section{Full-length Bm86 antigen}

In a comparative study performed in Texas, USA using Gavac $^{\circ}$ and a third party-derived formulation with Bm86, it was shown that efficacy against $R$. microplus was 27 and 50-65\% depending on the tick strain and adjuvant formulations used. However, vaccine efficacy was close to $100 \%$ against Rhipicephalus annulatus strains [8]. These results corroborate the hypothesis that strain differences affect the vaccine efficacy against $R$. microplus that is observed in different models/experiments [9]. It was emphasized by a number of presenters that vaccination with $\mathrm{Bm} 86$ is generally more effective against $R$. annulatus than against $R$. microplus [10]. In addition, cattle vaccinated with Bm86 were significantly protected against infestation with Rhipicephalus decoloratus, but not against Rhipicephalus appendiculatus [11]. Cattle vaccination with the $\mathrm{Bm} 86$ homologue of $R$. appendiculatus affected moulting from nymphs to adults from 95.5 to $89.3 \%$, a statistically significant effect [12]. The antigen (approximately $100 \mu \mathrm{g}$ protein per vaccine dose) was delivered subcutaneously as three separate inoculations at four week intervals in Montanide ISA $50 \mathrm{~V}$ (Seppic). Duration of immunity studies revealed that immunity induced with Bm86/Bm95 could last for 5-6 months, which has also been suggested after evaluation of the performance of the commercial vaccines TickGARD $^{\circ}$ and Gavac $^{\circ}$ over a period of 10 years in the field [6]. A single yearly booster vaccination is required to maintain this level of immunity. Gavac ${ }^{\circ}$ is commercially available in Latin American countries such as Cuba and Venezuela. TickGARD ${ }^{\circ}$ is not commercially available anymore but Alicja Lew-Tabor (The University of Queensland, Australia) mentioned that some stock is still available from Queensland University.

\section{Synthetic partial Bm86 antigen}

Joaquín Patarroyo (Universidade Federal de Viçosa, Brazil) presented results in which a vaccine based on three peptides of the Bm86 protein that differ only at two amino acid positions from the original Australian tick sequence is being used in the field in Brazil with $2 \mathrm{mg}$ of recombinant protein per dose with $1.5 \mathrm{mg}$ of saponin as adjuvant [13]. Cattle were vaccinated three times with a 30-day interval. Protection against tick infestation is $>80 \%$ reduction in engorged females. This study, which includes a number of nonvaccinated control farms where acaricide treatment is used to control tick populations, is on its third year and involves 15,000 cattle head in total.

\section{Ferritin 2(Fer-2)}

Fer-2 is a secreted molecule expressed in tick gut that functions in inter-tissue transport of non-heme iron originating from blood meal [14]. Vaccination of rabbits with Fer-2 from Ixodes ricinus expressed in E. coli provided protection against infestation with $I$. ricinus ticks (43 \% reduction in numbers of engorged female ticks; [15]. The Fer-2 homologue of $R$. microplus adjuvanted with Montanide ISA $50 \mathrm{~V}$ also induced protection when used as a vaccine in one experiment using cattle and $R$. microplus tick infestation. The protective effect was reflected in the number of engorged females being reduced by $30 \%$ and an additional effect on fertility resulting in a total efficacy of $64 \%$ on viable progeny [15]. Petr Kopáček (Institute of Parasitology, Biology Centre Czech Academy of Sciences, Czech Republic) presented a repeat study showing only partial protection affecting mainly oviposition and larval hatching and additional repeat studies are ongoing. Kopáček also emphasized the vaccination potential of other molecules possibly playing a role in tick heme and iron metabolic pathways of $I$. ricinus that were recently screened using RNA-interference (RNAi) and experimental vaccination of rabbits. However, none of the tested molecules surpassed Fer-2 in their impact on the tick development and reproduction. 


\section{Subolesin (SUB)}

SUB is an intracellular regulatory protein that is involved in signal transduction. José de la Fuente (SaBio, Instituto de Investigación en Recursos Cinegéticos, Spain) reported that cattle vaccination with SUB from $R$. microplus adjuvated with Montanide ISA $50 \mathrm{~V}$ reduced the numbers of engorged female ticks after infestation with R. microplus by $47 \%$ (60\% total efficacy considering the effect on oviposition and fertility; [5]). Similar results were found on vaccinated white-tailed deer. Additionally, partial protection has also been found for various hard and soft tick species and for other ectoparasites such as poultry red mite, mosquitoes and sand flies, hence its applicability in vaccines against haematophagous arthropods appears relatively wide [5]. Additionally, the effect of SUB vaccination on tick pathogen infection or transmission has been also documented, therefore suggesting its possible application to control vector infestations and pathogen infection [5]. A chimeric protein (Q38) that contains protective epitopes identified in SUB and the mosquito orthologue Akirin was used to vaccinate cattle (three intramuscular vaccinations; [16]). A single dose consisted of $100 \mu \mathrm{g}$ protein produced in E. coli and formulated with Montanide ISA $50 \mathrm{~V}$ adjuvant. Upon infestation with $R$. microplus, a reduction of $69 \%$ in the number of engorged females was observed. There was some additional effect on oviposition with $75 \%$ total efficacy.

\section{SILK antigen}

de la Fuente reported that the $R$. microplus SILK antigen was discovered for its role in tick-Anaplasma marginale interactions and used to vaccinate cattle with 3 intramuscular vaccinations [16]. A single dose consisted of $100 \mu \mathrm{g}$ protein produced in E. coli and formulated with Montanide ISA $50 \mathrm{~V}$ adjuvant. Upon infestation with $R$. microplus a reduction of $58 \%$ in the number of engorged females was observed. There was little additional effect on oviposition with $62 \%$ total efficacy.

\section{New vaccine antigen candidates}

A number of research groups are using reverse vaccinology or vaccinomics to discover new vaccine antigen candidates $[5,17]$. In order to limit the number of putative vaccine candidates, a number of selection criteria is being used such as antigens that evoke antibodies that interfere with coagulation or in vitro feeding of adult ticks. Some of these candidates were presented that have been tested in vaccination-challenge experiments. These new candidates include (a) antigens interfering with blood coagulation and digestion (TCX, reprolysins), (b) antigens interfering with in vitro tick feeding (mix of 6 peptides), and (c) antigens interfering with tick biology (Aquaporins). A Kunitz-type protein with unknown function that is found on the cell surface of $R$. microplus midgut cells (TCX), was identified as one of three protein-binding partners of Bm86 using a yeast two-hybrid assay [18]. Upon infestation with $R$. microplus larvae, a reduction of $74 \%$ in the number of engorged adult female ticks was found with a significant phenotype of "dandruff-like" nymphs and young adults. In studies using a combination of TCX and Bm86, the effect of Bm86 was increased four-fold. In addition, a combination of reprolysins, a group of metalloproteases from $R$. microplus [19] was used to vaccinate calves and induced approximately $60 \%$ reduction in the number of engorged females. All antigens were produced in E.coli and calves were vaccinated with $100 \mu \mathrm{g}$ antigen subcutaneously as three separate inoculations at four week intervals in Montanide ISA $50 \mathrm{~V}$.

Lew-Tabor reported that a mix of 6 peptides that were selected because of the fact that antibodies against those peptides reduced the proportion of ticks that successfully completed a blood meal in vitro was used to vaccinate cattle [17]. Upon infestation with $R$. microplus larvae, a reduction of $>85 \%$ in the number of engorged adult female ticks was found. When each of these peptides was evaluated individually, protection was usually much less (15-45\%), but trials are on-going testing the use of new formulations with these putative protective antigens.

During feeding ticks concentrate the blood meal in the midgut by removing water and excreting it back into the host as saliva. Aquaporins are membrane proteins that have been identified as playing a major role in this process. Out of three aquaporin genes identified in $R$. microplus, the potential use of Aquaporin 1 (RmAQP1) as a vaccine was recently tested. In two independent pen trials conducted in Brazil using Holstein calves vaccinated with Pichia-expressed recombinant RmAQP1 there was a high efficacy of 75 and $68 \%$, respectively, mainly due to the clear reduction in number of adult ticks that fed successfully [20]. The importance of Aquaporin 2 (RmAQP2) was demonstrated by RNAi mediated blocking of expression of this gene. There was a significant reduction in tick reproductive fitness and larval viability [21]. Interestingly, Glen A. Scoles (USDA, Washington State University, USA) reported that this effect appeared to be higher when the ticks were infected with Babesia bovis.

\section{Combined antigens}

In order to increase the level of protection by vaccination it has been suggested earlier to test combinations of antigens that each had shown to induce partial protection against tick infestations. A number of antigens have been used in combination with the Bm86 midgut protein with variable results. Theo Schetters (ProtActivity R\&D, The Netherlands) reported on the use of Bm86 combined with 
SUB. Cattle that were vaccinated with $100 \mu \mathrm{g}$ of Bm86 produced in P. pastoris and $50 \mu \mathrm{g}$ of SUB produced in $E$. coli in separate vaccine formulations in Montanide ISA $50 \mathrm{~V} 2$ adjuvant were almost completely protected against R. microplus infestation ( $>95 \%$ reduction in the number of engorged females; [22]). In vitro feeding studies using $R$. microplus larvae suggested that the effect is due to the synergistic activity of antibodies against each of the antigens. De la Fuente presented results using a chimeric protein composed of antigenic peptides from the $\mathrm{Bm} 95$ protein and the complete SUB protein fused to the $A$. marginale MSP1a protein to present the antigens on the surface of recombinant E. coli [23]. The bacterial membrane fraction containing $120 \mu \mathrm{g}$ of recombinant chimeric protein per dose adjuvanted with Montanide ISA50V2 was given three times through the intramuscular route and induced $60 \%$ reduction in $R$. microplus infestations and $91 \%$ efficacy considering the reduction of viable progeny in a preliminary trial in cattle.

\section{Efficacy models}

In order to evaluate the protective effects of cattle vaccination with tick antigens, a number of efficacy models are being used. Especially when using reverse vaccinology or vaccinomics, there is a need for efficient screening systems to select putative vaccine antigen candidates [5, 17]. Results obtained with different models were presented, and pros and cons were discussed.

\section{In vitro models}

Blocking of gene expression using RNAi can be used to discover genes that are essential for tick biology, however, this strategy does not specifically select for vaccine antigen candidates. Because protective immunity against tick infestation is mainly antibody mediated, a system that shows the protective activity of serum from experimental animals that were vaccinated with tick vaccine formulations is needed. Preferably, antibodies should be raised in immunized cattle but smaller laboratory animals can also be used during this phase of antigen screening. Protective activity of serum raised against Bm86 was demonstrated using tube feeding of adult $R$. microplus adult ticks and membrane feeding of $R$. microplus larvae [22]. The latter system is expected to become publically available this year, and has the advantage over capillary tube feeding of semi-engorged adult females that it allows more efficient screening of vaccine candidate antigens. The application of this system for nymphs and adult ticks needs to be validated. Importantly, in vitro feeding systems allow combining sera with different antigen specificity to discover synergetic effects of antibodies raised against these individual antigens. This combination increases the efficiency of antigen screening, and reduces the use of experimental animals and development costs. It should be realized, however, that identification of effective combinations of protective antigens by these methods does not cover the entire spectrum of putative protective antigens as some antigens will be missed.

\section{In vivo models}

Infestation of natural hosts with tick larvae is considered the best model to study the effects of vaccination on tick populations in the field. It was agreed at the meeting that vaccine efficacy should be expressed as reduction in the number of fully engorged adult ticks. There are essentially two techniques used: infestation at a confined space and whole body tick challenges. Confined tick infestations have been used for several tick species such as $R$. appendiculatus and $I$. ricinus using ear-wraps. However, as presented by Juan Mosqueda (Universidad Autónoma de Queretaro, Queretaro, Mexico), when studying ticks from the Boophilus group, nettings that are glued to the flank of the calf are usually applied, thus creating a patch where ticks feed. Confined infestations allow studying the effect of vaccination on different tick species on a single animal. This method has been used to study the effect of vaccination with Bm86 and SUB against $R$. microplus and $R$. annulatus [22]. It was generally agreed that whole body infestations reflect better a natural field infestation of cattle. For whole body infestations, larvae derived from tick colonies that are kept at the laboratory are put on the bovines at one or, preferably, more occasions at close intervals. In all cases, animals are restrained from grooming (e.g., with a short halter) for the duration of tick feeding to minimize grooming. Because ticks of the Boophilus group are one-host ticks, larvae will develop to nymphs and subsequently adults on the same animal. Fully engorged ticks that drop off from the animals in the third week after larval infestation must be recovered from the tray below the grid floor and purified from contaminating feces. Ticks can also be incubated to determine egg viability through the determination of percent emerging larvae. It should be clear that whole body tick infestations do not easily allow studying the effect of vaccination on different tick species, unless tick species determination using molecular biological techniques is additionally performed on ticks that are recovered from cattle. Schetters emphasized that when using whole body infestation, a minimal number of five cattle per experimental group is required to detect relevant statistical differences of at least $60 \%$ reduction in the number of engorged female ticks.

Ultimately, field experiments are required to study the effect of vaccination on tick infestation. The major problem is that the natural level of infestation cannot be controlled, which increases the number of animals per experimental group including a control group to reach a sufficient statistical power. It is important to evaluate the different methodologies used in field studies and standardize these in 
order to make comparison between different field trials. There was some debate on whether a confined challenge that forces the ticks to feed on a part of the body that is not the preferred site for feeding, might not reflect the natural situation. It was argued that the fact that in the natural situation there are usually fewer ticks feeding on the flank than for instance on the neck of the animals is likely due to the fact that cattle groom themselves, and remove ticks from those areas. An experimental trial that compares the development of graded numbers of ticks applied at a patch or applied as a whole body infestation was suggested. An important parameter is the proportion of larvae that develops to fully engorged ticks. The consortium should agree upon a minimal value to consider a vaccine formulation as efficacious for the control of cattle tick infestations.

\section{The way forward}

The meeting participants agreed on the pipeline for the development of effective vaccines for the control of cattle tick infestations (Fig. 2) and expressed their desire to continue this cattle tick vaccine initiative. It was suggested to formalize the formation of a Cattle Tick Vaccine Consortium (CATVAC). The consortium will be guided by a Steering Committee formed by Christine Maritz-Olivier, José de la Fuente and Theo Schetters (Chair) who will drive the project and take responsibility to realize the action points listed below.

\section{Standardization of tick vaccine research}

Standardization of tick vaccine research is of ultimate importance to allow comparison of results obtained by different research groups. A number of specific standards were defined and included (a) positive antigen control, (b) challenge model, (c) calculation of efficacy, and (d) correlate of protection. The meeting agreed to establish a positive antigen control for vaccine efficacy studies based on Bm86. This control preparation will be used in future efficacy trials. In addition, a standardized antibody test to determine the antibody titres against Bm86 will be developed and shared among participants. The meeting also agreed to define specific parameters for in vivo challenge models. Parameters that can be standardized are the age of the experimental animals, sex, breed, tick strain/colony and number of larvae used for infestation, the minimal proportion of larvae that develop to fully engorged adults, and the challenge model (whole body or patch infestation). In order to improve clarity about efficacy of tick vaccines, especially the way this is communicated in abstracts of scientific publications that are published on line, it was decided to always present the percentage reduction in the number of engorged females upon infestation with $R$. microplus larvae. In the case that also the effect of vaccination on oviposition and/or larval viability has been determined, this will be expressed as total efficacy, clearly stating which parameters were used to calculate this value (see Box 2 in [5]). Research should aim

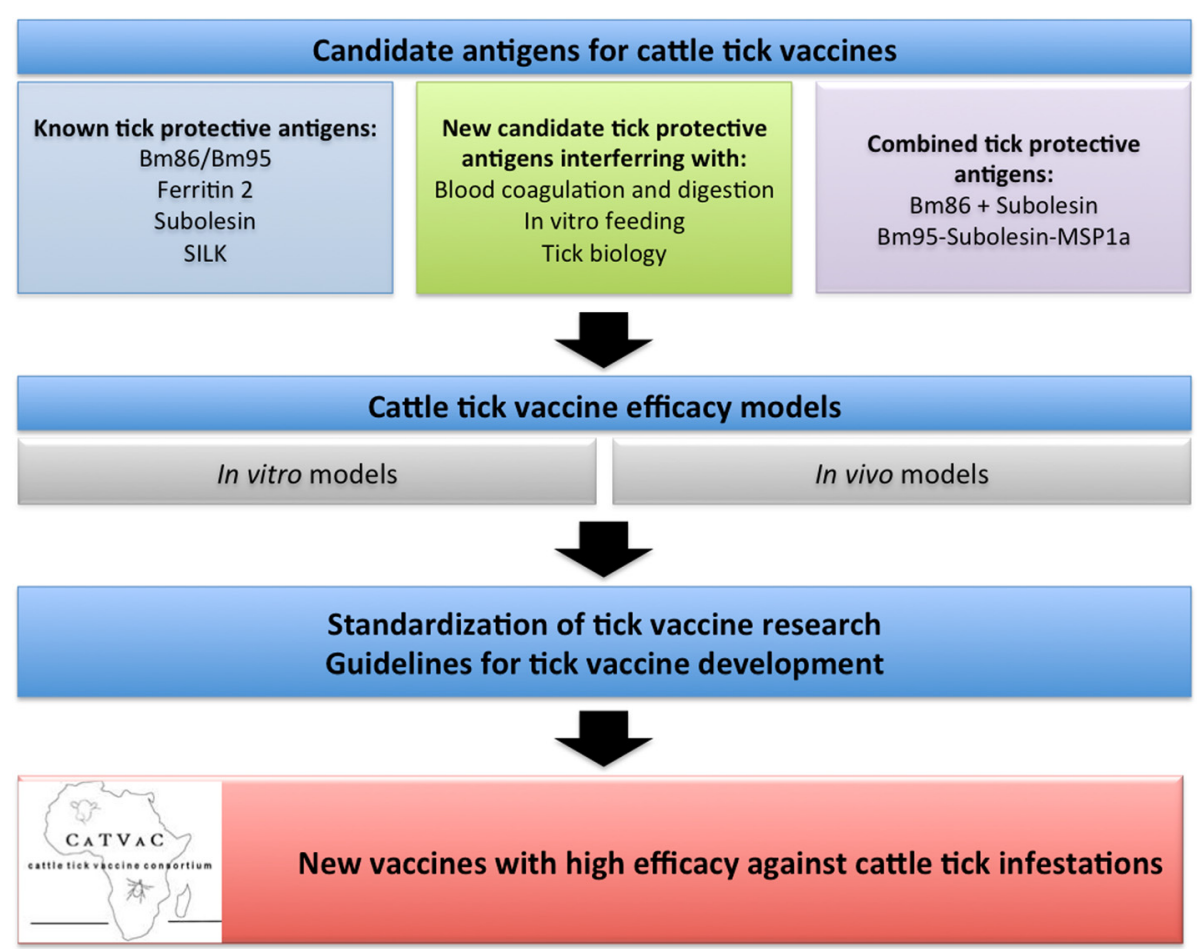

Fig. 2 Working pipeline proposed by CATVAC for development of effective vaccines for cattle tick control 
at defining a correlate of protection, which is a requirement for registration of the vaccine. Preferably, the correlate of protection is the antigen content or the antibody titer against the vaccine antigen(s). Such correlate of protection will be used to establish a potency test for the release of vaccine batches after quality control.

\section{Guidelines for tick vaccine development}

The standardization of tick vaccine research as described above forms the basis for the formulation of guidelines for tick vaccine development. These guidelines will be discussed with representatives of the World Association for the Advancement of Veterinary Parasitology (WAAVP) and submitted for publication in Veterinary Parasitology, the official organ of the WAAVP.

\section{Competing interests}

The authors have no competing interests relative to the work presented in this report.

\section{Authors' contributions}

TS and CMO conceived of and organized the meeting that generated the information provided in this report. TS, RB, MC, PK, ALT, CMO, RM, JM, JP, MRV, GAS and JF participated in the meeting and contributed to the update on the tick vaccine research and selection and evaluation of possible combinations of tick antigens that hold promise as an effective vaccine against cattle ticks, $R$. microplus using standardized experimental models. $T S, R B, M C, P K, A L T, C M O, R M, J M, J P, M R V, G A S$ and JF are members of the CATVAC. TS summarized discussions and recommendations from the meeting minutes. TS, PK and JF generated the initial draft of the manuscript. TS and JF were responsible for distribution of the manuscript to the co-authors and for revision in response to comments and for formatting the final manuscript version for submission. All authors read and approved the final version of the manuscript.

\section{Acknowledgements}

This meeting was supported by the Bill \& Melinda Gates Foundation. Special thanks to Emily Moran of the Gates foundation for helping to organize the meeting. The authors wish to express their gratitude to Dr. Peter Willadsen, Dr. Josephus Fourie (ClinVet International, Bloemfontein, South Africa) and Dr. Nick Juleff (Gates Foundation) for continuous support and advice. The opinions and assertions contained herein are those of the authors.

\footnotetext{
Author details

${ }^{1}$ ProtActivity R\&D, Cuijk, The Netherlands. ${ }^{2}$ ClinVet International, Bloemfontein, South-Africa. ${ }^{3}$ Tick Unit, International Livestock Research Institute ILRI, Nairobi, Kenya. ${ }^{4}$ Council for Scientific and Industrial Research (CSIR), Pretoria, Gauteng, South-Africa. Institute of Parasitology, Biology Centre Czech Academy of Sciences, Ceske Budejovice, Czech Republic. ${ }^{6}$ The University of Queensland, Queensland Alliance for Agriculture \& Food Innovation, St. Lucia, QLD, Australia. 'Murdoch University, Centre for Comparative Genomics, Perth, WA, Australia. ${ }^{8}$ The Genomics Research Institute, Department of Genetics, Faculty of Natural and Agricultural Sciences, University of Pretoria, Pretoria, South-Africa. ${ }^{9}$ Cattle Fever Tick Research Laboratory, Agricultural Research Service, United States Department of Agriculture, Edinburg, TX, USA. ${ }^{10}$ Facultad de Ciencias Naturales, Universidad Autónoma de Querétaro, Queretaro, Queretaro, Mexico. ${ }^{11}$ BIOAGRO/DVT Universidade Federal de Viçosa, Viçosa, MG, Brazil. ${ }^{12}$ Animal Disease Research Unit, Agricultural Research Service, United States Department of Agriculture, Washington State University, Pullman, Washington, USA. ${ }^{13}$ SaBio. Instituto de Investigación en Recursos Cinegéticos IREC CSIC-UCLM-JCCM, Ciudad Real, Spain. ${ }^{14}$ Department of Veterinary Pathobiology, Center for Veterinary Health Sciences, Oklahoma State University, Stillwater, OK, USA.
}

Received: 22 January 2016 Accepted: 14 February 2016 Published online: 24 February 2016

\section{References}

1. Grace D, Songe M, Knight-Jones T. Impact of neglected diseases on animal productivity and public health in Africa. In: 21st conference of the World Organisation for Animal Health (OIE) regional commission for Africa. Rabat: ILRI; 2015

2. Abbas RZ, Zaman MA, Colwell DD, Gilleard J, Iqbal Z. Acaricide resistance in cattle ticks and approaches to its management: the state of play. Vet Parasitol. 2014;203(1-2):6-20.

3. Adakal H, Biguezoton A, Zoungrana S, Courtin F, De Clercq EM, Madder M. Alarming spread of the Asian cattle tick Rhipicephalus microplus in West Africa-another three countries are affected: Burkina Faso, Mali and Togo. Exp Appl Acarol. 2013;61(3):383-6.

4. Allen JR, Humphreys SJ. Immunisation of guinea pigs and cattle against ticks. Nature. 1979;280(5722):491-3.

5. de la Fuente J, Contreras M. Tick vaccines: current status and future directions. Expert Rev Vaccines. 2015;14(10):1367-76.

6. de la Fuente J, Almazan C, Canales M, de la Lastra JM P, Kocan KM, Willadsen P. A ten-year review of commercial vaccine performance for control of tick infestations on cattle. Anim Health Res Rev. 2007:8(1):23-8.

7. Rand KN, Moore T, Sriskantha A, Spring K, Tellam R, Willadsen P, Cobon GS. Cloning and expression of a protective antigen from the cattle tick Boophilus microplus. Proc Natl Acad Sci U S A. 1989:86(24):9657-61.

8. Miller R, Estrada-Pena A, Almazan C, Allen A, Jory L, Yeater K, Messenger M, Ellis D, Perez de Leon AA. Exploring the use of an anti-tick vaccine as a tool for the integrated eradication of the cattle fever tick, Rhipicephalus (Boophilus) annulatus. Vaccine. 2012;30(38):5682-7.

9. Freeman JM, Davey RB, Kappmeyer LS, Kammlah DM, Olafson PU. Bm86 midgut protein sequence variation in South Texas cattle fever ticks. Parasit Vectors. 2010;3:101.

10. Pipano E, Alekceev E, Galker F, Fish L, Samish M, Shkap V. Immunity against Boophilus annulatus induced by the Bm86 (Tick-GARD) vaccine. Exp Appl Acarol. 2003;29(1-2):141-9.

11. Odongo D, Kamau L, Skilton R, Mwaura S, Nitsch C, Musoke A, Taracha E, Daubenberger C, Bishop R. Vaccination of cattle with TickGARD induces cross-reactive antibodies binding to conserved linear peptides of Bm86 homologues in Boophilus decoloratus. Vaccine. 2007;25(7):1287-96.

12. Olds C, Mwaura S, Crowder D, Odongo D, van Oers M, Owen J, Bishop R, Daubenberger C. Immunization of cattle with Ra86 impedes Rhipicephalus appendiculatus nymphal-to-adult molting. Ticks Tick Borne Dis. 2012;3(3):170-8.

13. Peconick AP, Sossai S, Girao FA, Rodrigues MQ, Souza ESCH, Guzman QF, Patarroyo VA, Vargas MI, Patarroyo JH. Synthetic vaccine (SBm7462) against the cattle tick Rhipicephalus (Boophilus) microplus: preservation of immunogenic determinants in different strains from South America. Exp Parasitol. 2008;119(1):37-43.

14. Hajdusek O, Sojka D, Kopacek P, Buresova V, Franta Z, Sauman I, Winzerling J, Grubhoffer L. Knockdown of proteins involved in iron metabolism limits tick reproduction and development. Proc Natl Acad Sci U S A. 2009;106(4):1033-8.

15. Hajdusek O, Almazan C, Loosova G, Villar M, Canales M, Grubhoffer L, Kopacek $P$, de la Fuente J. Characterization of ferritin 2 for the control of tick infestations. Vaccine. 2010;28(17):2993-8.

16. Merino O, Antunes S, Mosqueda J, Moreno-Cid JA, Perez de la Lastra JM, Rosario-Cruz R, Rodriguez S, Domingos A, de la Fuente J. Vaccination with proteins involved in tick-pathogen interactions reduces vector infestations and pathogen infection. Vaccine. 2013;31(49):5889-96.

17. Lew-Tabor AE, Bruyeres AG, Zhang B, Rodriguez Valle M. Rhipicephalus (Boophilus) microplus tick in vitro feeding methods for functional (dsRNA) and vaccine candidate (antibody) screening. Ticks Tick Borne Dis. 2014;5(5):500-10.

18. Louw E, van der Merwe NA, Neitz AW, Maritz-Olivier C. Evolution of the tissue factor pathway inhibitor-like Kunitz domain-containing protein family in Rhipicephalus microplus. Int J Parasitol. 2013;43(1):81-94.

19. Ali A, Parizi LF, Guizzo MG, Tirloni L, Seixas A, Vaz Ida S, Jr., Termignoni C. Immunoprotective potential of a Rhipicephalus (Boophilus) microplus metalloprotease. Vet Parasitol. 2015;207(1-2):107-14.

20. Hussein HE, Scoles GA, Ueti MW, Suarez CE, Adham FK, Guerrero FD, Bastos RG. Targeted silencing of the Aquaporin 2 gene of Rhipicephalus (Boophilus) microplus reduces tick fitness. Parasit Vectors. 2015;8:618. 
21. Guerrero FD, Andreotti R, Bendele KG, Cunha RC, Miller RJ, Yeater K, Perez de Leon AA. Rhipicephalus (Boophilus) microplus aquaporin as an effective vaccine antigen to protect against cattle tick infestations. Parasit Vectors. 2014;7:475.

22. Schetters TPM, Jansen T. Vaccine against Rhipicephalus ticks. Edited by WIPO, vol. WO2014154847 A12014. 2014.

23. Almazan C, Moreno-Cantu O, Moreno-Cid JA, Galindo RC, Canales M, Villar M, de la Fuente J. Control of tick infestations in cattle vaccinated with bacterial membranes containing surface-exposed tick protective antigens. Vaccine. 2012;30(2):265-72.

Submit your next manuscript to BioMed Central and we will help you at every step:

- We accept pre-submission inquiries

- Our selector tool helps you to find the most relevant journal

- We provide round the clock customer support

- Convenient online submission

- Thorough peer review

- Inclusion in PubMed and all major indexing services

- Maximum visibility for your research

Submit your manuscript at www.biomedcentral.com/submit
Biomed Central 